\title{
The mutational landscape of paroxysmal nocturnal hemoglobinuria revealed: new insights into clonal dominance
}

\author{
Stanley Chun-Wei Lee ${ }^{1}$ and Omar Abdel-Wahab ${ }^{1,2}$ \\ 'Human Oncology and Pathogenesis Program and 'Leukemia Service, Department of Medicine, Memorial Sloan Kettering Cancer Center, New York, New York, USA.
}

\begin{abstract}
Paroxysmal nocturnal hemoglobinuria (PNH) is a disorder of hematopoietic stem cells that has largely been considered a monogenic disorder due to acquisition of mutations in the gene encoding PICA, which is required for glycosylphosphatidylinositol (CPI) anchor biosynthesis. In this issue of the $J C I$, Shen et al. discovered that PNH is in fact a complex genetic disorder orchestrated by many genetic alterations in addition to PIGA mutations. Some of these mutations predate the acquisition of PICA mutations, while others occur later. Surprisingly, this work indicates that PNH has a clonal evolution and architecture strikingly similar to that of other myeloid neoplasms, highlighting a potentially broader mechanism of disease pathogenesis in this disorder.
\end{abstract}

\section{Paroxysmal nocturnal hemoglobinuria: a monogenic disorder?}

Paroxysmal nocturnal hemoglobinuria (PNH) is an acquired clonal disorder that arises from hematopoietic stem cells (HSCs) and affects 1-2 people per million, with a median age at diagnosis of 35 to 40 years. Without treatment, $\mathrm{PNH}$ is a life-threatening disease characterized by intravascular hemolysis, pancytopenia, and venous thrombosis. Although $\mathrm{PNH}$ is rare, there is extensive knowledge regarding its pathophysiology and underlying molecular defects. In fact, PNH serves as a paragon of success for molecular medicine, as a series of elegant translational studies identified the precise mechanistic basis for many aspects of the disease, resulting in a remarkably efficacious mechanism-based therapy.

$\mathrm{PNH}$ occurs as a consequence of acquired somatic mutations in phosphatidylinositol glycan class A (PIGA) (1), located on the short arm of the X chromosome (Xp22.1). PIGA encodes an enzyme that is critical in the first step of the biosynthesis of a class of glycolipid proteins known as the glycosylphosphatidylinositol anchor proteins (GPI-APs) (2-4). GPI modifications function to anchor a number of membrane proteins to the surface of hematopoietic cells. Among the GPI-APs absent from the surface of hematopoietic cells in $\mathrm{PNH}$ patients are the complement inhibitors CD55 and CD59, the loss of which confer heightened sensitivity of rbc to complement-induced hemolysis. Likewise, activation of complement due to loss of CD55 and CD59 in PNH patients explains the propensity for thrombosis in this disorder.

$\mathrm{PNH}$ has largely been considered a monogenic disorder, with many disease manifestations fully explained by the presence of mutations in PIGA. However, there is one critical question regarding $\mathrm{PNH}$ that has yet to be fully explained despite several decades of research: What is the mechanistic basis for the clonal stem cell advantage of PIGA-mutant cells? It is widely understood that $\mathrm{PNH}$ is a clonal

Related Article: p. 4529

Conflict of interest: The authors have declared that no conflict of interest exists

Reference information: / Clin Invest. 2014;124(10):4227-4230. doi:10.1172/JCI77984.

disease that originates from HSCs, as evidenced by the repeated observation that PNH patients have PIGA mutations in cells of multiple lineages, including myeloid, erythroid, and lymphoid cells, though an explanation of how mutant stem cells harboring PIGA mutations sustain clonal expansion over time has remained elusive.

\section{Deep sequencing reveals} collective mutations in $\mathrm{PNH}$

In this issue, Shen et al. present a comprehensive description of the constellation of somatic mutations present in PNH clones (5). Shen and colleagues performed whole-exome sequencing (WES) in $12 \mathrm{PNH}$ patients and targeted deep sequencing in 36 additional patients on 61 genes that are commonly mutated in myeloid malignancies. Sequencing was performed on DNA extracted from CD59hematopoietic cells (indicative of $\mathrm{PNH}$ ), with $\mathrm{CD} 59^{+}$cells serving as a premutation reference. Shen et al. have provided an extensive report using unbiased sequencing on a large patient cohort to examine the genomic landscape of PNH. They revealed that many mutations, most of which have not been previously associated with $\mathrm{PNH}$, occur in tandem with PIGA mutations. Moreover, Shen et al. found that these additional mutations arise either as a sub-clone within the PIGA-mutant cell population or as an initial genetic event prior to the acquisition of the PIGA mutation (Figure 1).

One of the key findings by Shen et al. is the revelation that $\mathrm{PNH}$ clones with multiple mutations were present at substantially higher frequencies than those of clones with only mutations in PIGA (5). This finding implies that the presence of additional mutations confers an intrinsic growth advantage for PIGA-mutant cells. The clonal dominance in $\mathrm{PNH}$ has previously been speculated to occur due to either an intrinsic growth advantage of PIGA-mu- 


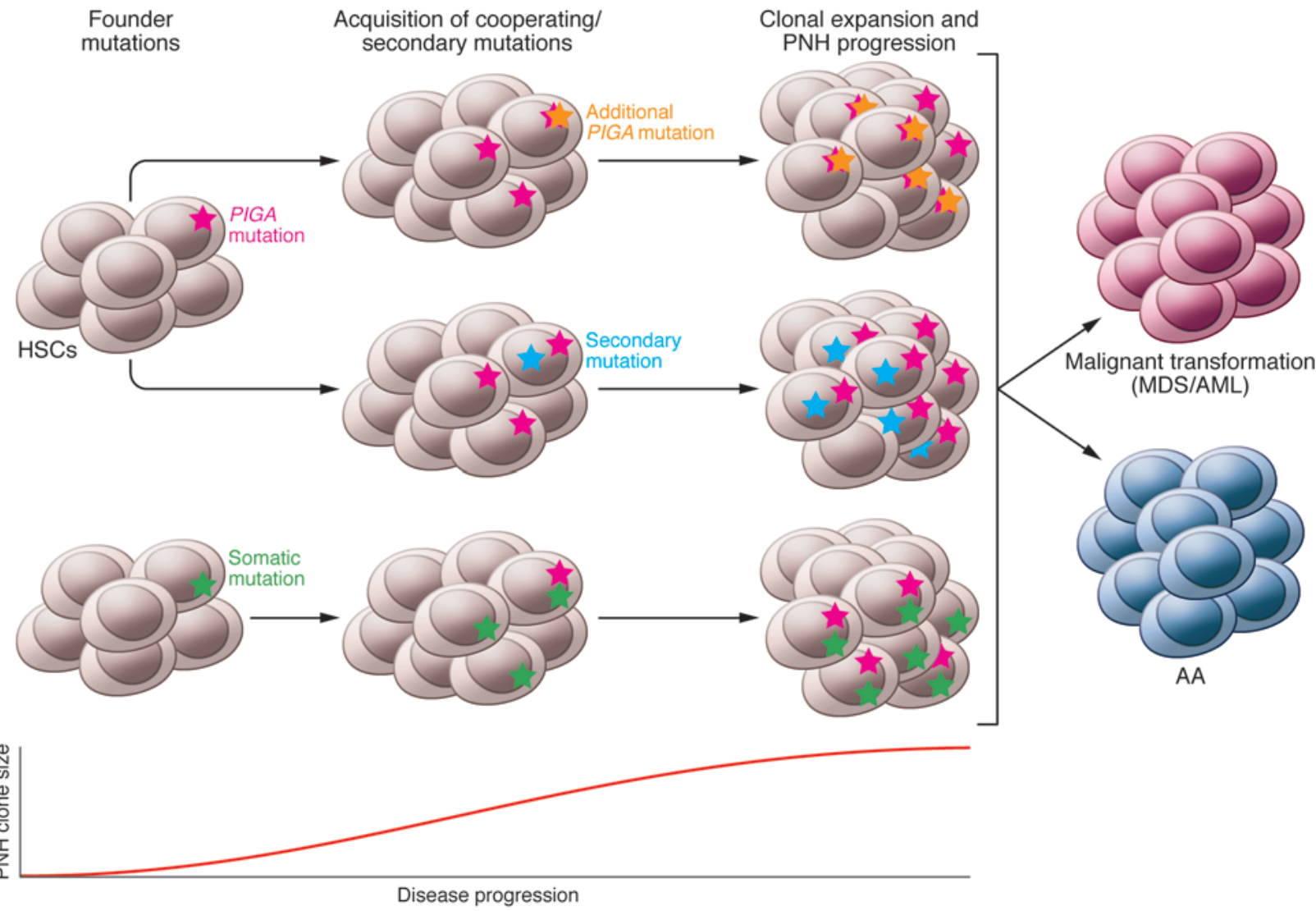

Figure 1. Clonal architecture and evolution of PNH. PNH arises from an initial mutation in PICA (orange) or in a different founder gene (green) in HSCs. Over time, the acquisition of additional cooperating lesions, which can include additional mutations in PICA (yellow) or other genes (blue) (e.g., TET2, SUZ12, JAK2, and U2AF1), in addition to the founder mutation, adds complexity to the clonal architecture and confers a growth advantage and subsequent progression into overt PNH. On rare occasions, PNH clones can transform into myeloid malignancies such as MDS and acute AML, or into AA. Future work to identify the complement of events responsible for the transformation of PNH into MDS or AML will be enlightening. Moreover, it will be important to determine whether AA harbors a genetic complexity and clonal architecture similar to that of PNH.

tant cells (the "relative growth advantage hypothesis") and/or to cell-extrinsic factors that might provide a relative growth advantage for PIGA-mutant cells (the socalled "immune escape hypothesis"). The "relative growth advantage hypothesis" posits that the PIGA mutation alone is essential, but not sufficient, to cause $\mathrm{PNH}$. Consistent with this hypothesis, rare PIGA mutations have been found in a very small proportion of HSCs in healthy individuals who do not have PNH. At the same time, the fact that some $\mathrm{PNH}$ patients have been found to harbor several different PIGA mutations simultaneously supports the notion that the presence of PIGA mutations alone underlies clonal advantage. Given these discrepancies, several different Piga-knockout mice have been generated in attempts to definitively establish the causal role of PIGA mutations in HSC function. Detailed analyses of these models have collectively demonstrated that Piga $^{-/-}$
HSCs do not have an absolute proliferative advantage over WT HSCs, supporting the notion that additional independent factors are required for the expansion of mutant clones (6-10). Furthermore, the studies in Piga-mutant mice support the possibility that collaborating mutations are required to drive $\mathrm{PNH}$, similar to mechanisms of oncogenic transformation. Indeed, some candidate secondary mutations have been described, albeit in a very small patient pool, including mutations in $H M G 2 A$, $N R A S$, and $J A K 2$, as well as rare cytogenetic alterations, all of which lend support to the requirements for secondary collaborating events in $\mathrm{PNH}$ pathogenesis (11-14).

The genetic evidence that an intrinsic clonal advantage of PNH cells is due to collaboration between PIGA mutations and additional somatic mutations still does not eliminate the possibility that cell-extrinsic factors contribute in the clonal dominance of PNH. The notion of a cell-nonauton- omous aspect of PNH clonal dominance came from the observation that $\mathrm{PNH}$ frequently co-occurs with immune-mediated aplastic anemia (AA), in which hematopoiesis is compromised due to suppression of a proportion of HSCs. In addition to the absence of CD55 and CD59, the lack of GPIAPs also renders PNH immune cells deficient in a number of cell-surface proteins that normally mediate immune responses and activate $\mathrm{T}$ cell receptor signaling. The “immune escape hypothesis" of $\mathrm{PNH}$ posits that the lack of immune-mediated recognition provides PIGA-deficient HSCs an extrinsic survival advantage, allowing escape from effector immune cell attack. Gene expression profiling of GPI-normal $\mathrm{CD} 4^{+}$cells from $\mathrm{PNH}$ patients showed upregulation of genes involved in immune responses, while expression patterns of GPI-deficient CD34+ cells were remarkably similar to those found in healthy donors. More interestingly, expression of 
proliferation-associated genes was similar in CD $34^{+}$HSCs isolated from both normal and PNH clones (15). Another study evaluated $\mathrm{CD} 34^{+}$cells from $\mathrm{PNH}$ patients that were purified based on PIGA mutation status and revealed that $\mathrm{CD}^{-} 9^{-} \mathrm{CD} 34^{+}$(indicative of $\mathrm{PNH}$ ) clones proliferated normally ex vivo compared with healthy $\mathrm{CD} 34^{+}$ HSCs, while CD59+ CD $34^{+}$clones had proliferation defects $(16,17)$. Together, these findings highlight an important role for cell-extrinsic factors in dictating the clonal expansion of PNH cells.

In addition to identifying mutations that co-occur with the loss of PIGA, Shen et al. also performed clonogenic assays, bacterial subcloning, and quantitative mutational analyses to assess the temporal acquisition of mutations in $\mathrm{PNH}$ (5). Intriguingly, in the majority of $\mathrm{PNH}$ patients, additional mutations were present prior to acquisition of the PIGA mutation, suggesting either a secondary role for PIGA mutations in PNH pathogenesis or that these other mutant clones are predisposed to developing PIGA mutations and subsequently PNH. Notably, some of the mutations identified by Shen et al. are frequently found in patients with myeloid malignancies such as myelodysplastic syndromes (MDS) and acute myeloid leukemia (AML). These myeloid malignancyassociated genes include tet methylcytosine dioxygenase 2 (TET2), zinc finger SUZ12, transcription factor ASXL1, BCL6 corepressor (BCOR), JAK2, and U2 small nuclear RNA auxiliary factor 1 (U2AF1). These findings by Shen et al. reveal that $\mathrm{PNH}$, a disease considered to be benign, shares genetic alterations and clonal composition similar to those of overtly malignant myeloid leukemias.

\section{Conclusions and future directions}

The observations by Shen and colleagues raise immediate questions regarding the functional consequences of these genetic lesions in hematopoiesis and their impact on the pathophysiology of PNH (5). For instance, what are the roles of these cooccurring mutations in $\mathrm{PNH}$ and how do they influence HSC function and differentiation? While it is unsatisfactory to hypothesize that these mutations that otherwise drive MDS play a passenger role in the presence of PIGA mutation, one can only infer that mutations occurring in clones with existing PIGA mutations possess the ability to alter the MDS disease phenotype. Indeed, most $\mathrm{PNH}$ patients with PIGA mutations and additional mutations in TET2, SUZ12, ASXL1, JAK2, or $U 2 A F 1$ had no signs of progressing to MDS or AML. Development of multiple murine models that harbor the mutations found to coexist with PIGA mutations will provide precious reagents to formally demonstrate the genetic interactions between PIGA deficiency and the mutations identified by Shen and colleagues.

Despite the extensive work performed on $\mathrm{PNH}$, there are still many interesting questions that remain unanswered. One key clinical feature in $\mathrm{PNH}$ patients is the defective hematopoietic function of HSCs without PIGA mutations, a phenomenon thought to be primarily driven by autoimmunity, as observed in AA patients. While it is apparent that extrinsic factors are predominantly involved in the destruction of HSCs and the bone marrow microenvironment, one cannot exclude the possibility that there are cell-intrinsic abnormalities in HSCs with WT PIGA from PNH patients. Characterizing the genetic differences between $\mathrm{CD}^{\circ} 9^{+}$and $\mathrm{CD}^{-} 9^{-} \mathrm{HSCs}$ from PNH patients will be a fascinating next step.

Now that we have gained a glimpse and new insight into the complex genomic landscape of $\mathrm{PNH}$, a benign disease that shares a clonal architecture and disease evolution similar to that seen in malignant neoplasms, it will be important to determine whether a similar mutation acquisition occurs in related hematological disorders. For example, ongoing genome-wide mutation discovery efforts in AA, an autoimmune disease of the bone marrow in which patients harbor $\mathrm{PNH}$, will be very informative in this regard (18). Moreover, a better understanding of the genetic events responsible for the transformation of PNH into malignant neoplasms, including MDS and AML, will hopefully continue the legacy of molecular discovery and therapeutic advances in $\mathrm{PNH}$.

\section{Acknowledgments}

O. Abdel-Wahab is supported by an $\mathrm{NIH} \mathrm{K08} \mathrm{Clinical} \mathrm{Investigator} \mathrm{Award}$ (1K08CA160647-01); a US Department of Defense Postdoctoral Fellow Award in Bone Marrow Failure Research (W81XWH-12-1-0041); the Josie Robert- son Investigator Program; and a Damon Runyon Clinical Investigator Award with support from the Evans Foundation.

Address correspondence to: Omar AbdelWahab, Human Oncology and Pathogenesis Program and Leukemia Service, Department of Medicine, Memorial Sloan Kettering Cancer Center, New York, New York 10065, USA. Phone: 646.888.3487; E-mail: abdelwao@mskcc.org.

1. Takeda J, et al. Deficiency of the GPI anchor caused by a somatic mutation of the PIG-A gene in paroxysmal nocturnal hemoglobinuria. Cell. 1993;73(4):703-711.

2. Armstrong $\mathrm{C}$, et al. Affected paroxysmal nocturnal hemoglobinuria $\mathrm{T}$ lymphocytes harbor a common defect in assembly of $\mathrm{N}$-acetyl-D-glucosamine inositol phospholipid corresponding to that in class A Thy-1- murine lymphoma mutants. J Biol Chem. 1992;267(35):25347-25351.

3. Hirose S, et al. Synthesis of mannosylglucosaminylinositol phospholipids in normal but not paroxysmal nocturnal hemoglobinuria cells. Proc Natl Acad Sci U S A. 1992;89(13):6025-6029.

4. Takahashi M, et al. Deficient biosynthesis of $\mathrm{N}$-acetylglucosaminyl-phosphatidylinositol, the first intermediate of glycosyl phosphatidylinositol anchor biosynthesis, in cell lines established from patients with paroxysmal nocturnal hemoglobinuria. J Exp Med. 1993;177(2):517-521.

5 . Shen W, et al. Deep sequencing reveals stepwise mutation acquisition in paroxysmal nocturnal hemoglobinuria. J Clin Invest. 2014;124(10):4529-4538.

6. Jasinski M, Keller P, Fujiwara Y, Orkin SH, Bessler M. GATA1-Cre mediates Piga gene inactivation in the erythroid/megakaryocytic lineage and leads to circulating red cells with a partial deficiency in glycosyl phosphatidylinositol-linked proteins (paroxysmal nocturnal hemoglobinuria type II cells). Blood. 2001;98(7):2248-2255.

7. Keller P, et al. FES-Cre targets phosphatidylinositol glycan class A (PIGA) inactivation to hematopoietic stem cells in the bone marrow. JExp Med. 2001;194(5):581-589.

8. Murakami Y, Kinoshita T, Maeda Y, Nakano T, Kosaka H, Takeda J. Different roles of glycosylphosphatidylinositol in various hematopoietic cells as revealed by a mouse model of paroxysmal nocturnal hemoglobinuria. Blood. 1999;94(9):2963-2970.

9. Rosti V, Tremml G, Soares V, Pandolfi PP, Luzzatto L, Bessler M. Murine embryonic stem cells without pig-a gene activity are competent for hematopoiesis with the $\mathrm{PNH}$ phenotype but not for clonal expansion. J Clin Invest. 1997;100(5):1028-1036.

10. Tremml G, et al. Increased sensitivity to complement and a decreased red blood cell life span in mice mosaic for a nonfunctional Piga gene. Blood. 1999;94(9):2945-2954.

11. Inoue N, et al. Molecular basis of clonal expansion of hematopoiesis in 2 patients with paroxysmal nocturnal hemoglobinuria (PNH). Blood. 
2006;108(13):4232-4236.

12. Mortazavi Y, Tooze JA, Gordon-Smith EC, Rutherford TR. N-RAS gene mutation in patients with aplastic anemia and aplastic anemia/paroxysmal nocturnal hemoglobinuria during evolution to clonal disease. Blood. 2000;95(2):646-650.

13. O'Keefe CL, et al. Deletions of Xp22.2 including PIG-A locus lead to paroxysmal nocturnal hemoglobinuria. Leukemia. 2011;25(2):379-382.

14. Sugimori C, et al. Paroxysmal nocturnal hemoglobinuria and concurrent JAK2(V617F) muta- tion. Blood Cancer J. 2012;2(3):e63.

15. Chen G, Zeng W, Maciejewski JP, Kcyvanfar K, Billings EM, Young NS. Differential gene expression in hematopoietic progenitors from paroxysmal nocturnal hemoglobinuria patients reveals an apoptosis/immune response in 'normal' phenotype cells. Leukemia. 2005;19(5):862-868.

16. Chen R, et al. Impaired growth and elevated fas receptor expression in PIGA(+) stem cells in primary paroxysmal nocturnal hemoglobinuria. JClin Invest. 2000;106(5):689-696.
17. Maciejewski JP, Sloand EM, Sato T, Anderson $\mathrm{S}$, Young NS. Impaired hematopoiesis in paroxysmal nocturnal hemoglobinuria/aplastic anemia is not associated with a selective proliferative defect in the glycosylphosphatidylinositol-anchored protein-deficient clone. Blood. 1997;89(4):1173-1181.

18. Yoshizato T, et al. Spectrum of genetic alterations in acquired aplastic anemia. Presented at: 2013 ASH Annual Meeting and Exposition; December 7-10, 2013; New Orleans, Louisiana, USA. 